Background Attitudes towards sex and relationships influence sexual behaviour, the wellbeing of those maraginalised, and provision and use of services. Some countries conduct repeated surveys on sexual attitudes, but no longitudinal studies have investigated individuals' changes in opinion over time. We have quantified consistency and change in acceptance of same-sex partnerships between ages 26 and 38 years, in New Zealand.

Methods Members of the Dunedin Study birth cohort answered computer-presented questions on their opinions about sexual and reproductive topics, including acceptability of same-sex partnerships, using items from the UK's National Survey of Sexual Attitudes and Lifestyles (Natsal-1). Opinions from the age 26 and age 38 assessments were compared for consistency and change, and associated characteristics.

Results Response level was $>90 \%$ at each assessment $(\mathrm{n}=966$; $\mathrm{n}=936$ ). The distribution of women's opinions was similar for sex between men and sex between women, and liberalised with age. Men's acceptance of sex between men was lower, and did not increase with age. At age 38 vs $26,38 \%$ vs $38 \%$ of men and $58 \%$ vs $54 \%$ of women reported sex between two men was 'rarely wrong/not wrong at all'. For sex between women, 58\% vs $55 \%$ of men and $60 \%$ vs $57 \%$ of women reported this level of acceptance. Although aggregate changes were small, at an individual level $42 \%$ of men and 35\% of women changed their opinion about sex between men, and about sex between women. For men and women, consistent acceptance of same-sex partnerships was associated having some same-sex experience, and more education.

Conclusions A fairly stable level of acceptance of same-sex sexual behaviour over 12 years was seen in this cohort but it obscures considerable flux in opinions among individuals. Better understanding of what influences opinions to become more or less liberal with age could inform health promotion.

Disclosure of interest This work was supported by the Health Research Council of New Zealand [12/1086]. The authors have no conflicts of interest.

\section{P04.15 SEXUAL BEHAVIOUR, STI TESTING AND DIAGNOSIS DOWN UNDER: FINDINGS FROM THE SECOND AUSTRALIAN STUDY OF HEALTH AND RELATIONSHIPS (ASHR2)}

${ }^{1}$ A Yeung ${ }^{*},{ }^{1} \mathrm{~T}$ Caruana, ${ }^{2} \mathrm{~A}$ Grulich, ${ }^{3} \mathrm{R}$ de Visser, ${ }^{4} \mathrm{C}$ Rissel, ${ }^{4} \mathrm{~J}$ Simpson, ${ }^{1} \mathrm{~J}$ Richters. ${ }^{1}$ School of Public Health and Community Medicine, University of New South UK; ${ }^{2} T$ The Kirby Institute, University of New South UK; ${ }^{3}$ School of Psychology, University of Sussex; ${ }^{4}$ Sydney School of Public Health, University of Sydney

\subsection{6/sextrans-2015-052270.269}

Background In recent years, the number of sexually transmissible infections (STIs) has skyrocketed in Australia and cause a considerable burden on public health. The association between STIs and sexual behaviour is well-established in high-risk populations but information about the general population is less well-known. ASHR2 provides population-based data about the sexual health and practices of Australians. This analysis examines the associations between STIs and sexual behaviour.

Methods Computer-assisted telephone interviews were conducted with a random sample of the Australian population aged 16-69. Participants were surveyed about their sexual behaviour and experiences with STIs. Responses were weighted based on study design, location, and the age and sex distribution of Australia. Univariate logistic regression was used to determine the associations between sexual behaviour, testing history and diagnosis.

Results A total of 20094 men and women were interviewed, with an overall participation rate of $66.2 \%$. A higher proportion of women $(17.3 \%)$ were tested than men $(13.2 \%)$ in the past year. Men and women were more likely to have been tested in the past year if they identified as bisexual (or homosexual for men), were of Aboriginal and/or Torres Strait Islander origin, had $>1$ partner in the past year, had a previous STI and had ever injected drugs. In the year prior to being interviewed, $1.1 \%$ of men and $2.7 \%$ of women reported having an STI. Diagnosis was associated with having $>1$ partner in the past year, sex work as a worker and having ever injected drugs for both men and women. For men, diagnosis was also associated with sex work as a client $(\mathrm{OR}=3.14$ [95\% CI: $1.78-5.56])$ while diagnosis in women was associated with receiving sex education at school (OR $=1.59[95 \%$ CI: $1.14-2.21])$.

Conclusion Australian women are tested more frequently for STIs and are diagnosed more frequently despite sharing similar associations for STIs as men.

Disclosure of interest statement No conflict of interest.

\section{P04.16 "IT OPENED MY EYES" - EXAMINING THE IMPACT OF THE AUSTRALIAN CHLAMYDIA CONTROL EFFECTIVENESS PILOT (ACCEPT) ON CHLAMYDIA TESTING PRACTICES OF GENERAL PRACTITIONERS}

${ }^{1}$ A Yeung* ${ }^{2}{ }^{2}$ H Hocking, ${ }^{2} \mathrm{~A}$ Vaisey, ${ }^{3} \mathrm{R}$ Lorch, ${ }^{3} \mathrm{R}$ Guy, ${ }^{4,5} \mathrm{CK}$ Fairley, ${ }^{3} \mathrm{~K}$ Smith, ${ }^{6} \mathrm{~J}$ Imrie, ${ }^{3,7} \mathrm{~B}$ Donovan, ${ }^{8} \mathrm{~J}$ Gunn, ${ }^{8} \mathrm{M}$ Temple-Smith, on behalf of the ACCEPt Consortium. ${ }^{1}$ School of Public Health and Community Medicine, University of New South UK; ${ }^{2}$ Centre for Epidemiology and Biostatistics, Melbourne School of Population and Global Health, University of Melbourne; ${ }^{3}$ The Kirby Institute, University of New South UK; ${ }^{4}$ Melbourne Sexual Health Centre; ${ }^{5}$ Central Clinical School, Monash University; ${ }^{6}$ UCL Centre for Sexual Health and HIV Research, University College London; ' Sydney Sexual Health Centre; ${ }^{8}$ General Practice and Primary Health Care Academic Centre, University of Melbourne

\subsection{6/sextrans-2015-052270.270}

Background In Australia, despite dramatic increases in chlamydia notifications and recommendations that young people be tested annually, testing rates in general practice remain low. ACCEPt is a randomised controlled trial to evaluate the impact of chlamydia testing on prevalence. General practitioners (GPs) in the intervention arm received regular feedback on testing performance, visual reminders and incentive payments to facilitate increased testing. To assess the acceptability of the intervention, participating GPs were interviewed at baseline and midpoint to investigate their chlamydia management practices and their opinions about the intervention.

Methods Semi-structured interviews were conducted with purposively sampled GPs prior to randomization (baseline) and after one year in the intervention (midpoint) until saturation was reached. Data were analysed both thematically and using Normalisation Process Theory (NPT), a framework that explains how complex interventions can be successfully implemented, embedded and integrated in institutional settings. Emerging themes from each timepoint were compared and interpreted within the NPT framework.

Results A total of 44 GPs at baseline and 24 GPs at midpoint were interviewed. The ACCEPt intervention increased GPs' comfort and ease in offering a test, and were associated with a shift in attitudes from symptom-based testing to age-based testing. Many GPs highlighted that feedback, a key aspect to embedding the intervention, was a major driver of increased testing. The 
intervention was gradually implemented over time through increasing awareness and engagement with education and exposure to the project. Logistical issues, such as remembering to offer a test, were overcome with practical facilitators like computer alerts. However, integration was limited as not all GPs utilised the intervention components or other clinic staff to increase testing because of restrictions in clinic structure.

Conclusion GPs reported that the ACCEPt intervention and its implementation within the NPT framework have been effective at facilitating chlamydia testing in general practice.

Disclosure of interest statement ACCEPt was commissioned and funded by the Australian Government Department of Health and Ageing. Additional funding has been received from the National Health and Medical Research Council, the Victorian Department of Health and NSW Health.

\section{P04.17 INTRAVAGINAL PRACTICES AMONG RURAL MALAWIAN WOMEN}

${ }^{1}$ A Esber*, ${ }^{2}$ A Norris Turner, ${ }^{1}$ A Norris. ${ }^{1}$ Division of Epidemiology, College of Public Health, The Ohio State University; ${ }^{2}$ Divison of Infectious Diseases, College of Medicine, The Ohio State University

10.1136/sextrans-2015-052270.271

Introduction Intravaginal practices (IVP) are highly prevalent and commonly performed in many countries for a variety of purposes related to genital health, personal hygiene, and sexual pleasure. However, IVP may also have harmful side effects. Previous research supports an association between IVP and bacterial vaginosis and HIV. Our objective is to understand the prevalence and motivations for IVP among rural Malawian women participating in a community survey on sexual and reproductive health.

Methods We used baseline survey data from a community-based cohort study conducted among 650 women in rural Lilongwe District, Malawi. Participants answered questions assessing frequency of use for different types of IVP and reasons for performing IVP.

Results Most women reported at least some experience with IVP in the past 30 days: $88 \%$ reported internal vaginal cleansing with water, $87 \%$ reported cleansing with soap and water, and $84 \%$ reported inserting cotton, cloth or tissue. Only 5\% of women reported no IVP; most (60\%) reported at least three practices. Approximately half of women reported very frequent engagement in at least one type of IVP: among those who inserted cotton, cloth or tissue, $43 \%$ did so more than once a day; among those who cleansed internally with soap and water, $51 \%$ did so more than once a day. Among IVP engagers, 57\% reported sexual- and hygiene-related reasons, $36 \%$ only hygiene reasons, $4 \%$ neither, and 3\% only sexually-related motivations. We found no significant association between motivations for IVP (for hygiene vs. sexual reasons) and frequency of IVP.

Conclusion Intravaginal practices are highly prevalent and frequently performed among these rural Malawian women. Women's motivations for IVP were not associated with IVP frequency, and therefore IVP cessation programs targeted to motivations may be unsuccessful in this population. The next phase of this research program will investigate the association between IVP and STI prevalence.

Disclosure of interest statement The authors have no conflicts of interest or financial disclosures to report.
P04.18 ARE INTRAVAGINAL PRACTICES ASSOCIATED WITH PRECANCEROUS LESIONS AND HPV INFECTION?

${ }^{1}$ A Esber*, ${ }^{1}$ A Norris, ${ }^{2}$ AN Turner. ${ }^{1}$ Division of Epidemiology, College of Public Health, The Ohio State University; ${ }^{2}$ Divison of Infectious Diseases, College of Medicine, The Ohio State University

\subsection{6/sextrans-2015-052270.272}

Introduction Many women engage in intravaginal practices (IVP) to improve genital hygiene and sexual pleasure. However, IVP can disrupt the genital mucosa, possibly increasing acquisition risk of HIV and the reproductive tract infection bacterial vaginosis. Limited prior research also suggests an association between IVP and HPV. In this analysis, we examine associations between IVP, precancerous lesions and high risk HPV (hr-HPV).

Methods At a rural clinic in Lilongwe District, Malawi, we began enrolling women in a cross-sectional study in January 2015. As of end of March, 96 women have been enrolled and partial results are available. Enrollment is expected to conclude by May 2015; hr-HPV testing to be complete by June 2015. Eligible women are aged 18 and older and presenting at the clinic with genitourinary symptoms. All women undergo visual inspection of the cervix with acetic acid (VIA) and clinician-collected cervical sampling for hr-HPV testing. Women also answer a questionnaire capturing the type and frequency of IVP.

Results IVP were commonly reported among participants: 97\% reported cleansing the vagina with cotton, cloth or tissue; $44 \%$ cleansed with soap and water; and $8 \%$ inserted alum or other powder, herbs, leaves, castor oil or traditional products. IVP was also very frequent, with $85 \%$ of women reporting at least one practice daily. Twelve women (15\%) had abnormal lesions identified during VIA. We observed no significant association between frequency or type of IVP and the presence of abnormal lesions.

Conclusion Our data confirm that intravaginal practices are common in Malawi among care-seeking women. As our sample size increases, any associations will become more precise. If IVP is associated with abnormal cervical lesions or hr-HPV prevalence, these practices could represent a modifiable risk factor to target in future health interventions in this region.

Disclosure of interest statement The authors have no conflicts of interest or financial disclosures to report.

\section{P04.19 ALCOHOL INVOLVEMENT IN SEXUAL BEHAVIOUR AND ADVERSE SEXUAL HEALTH OUTCOMES IN EARLY MIDDLE AGE}

JL Connor ${ }^{*}, \mathrm{R}$ Kydd, N Dickson. Department of Preventive and Social Medicine, University of Otago, Dunedin, New Zealand

10.1136/sextrans-2015-052270.273

Introduction Research on alcohol and sexual behaviour has focused on young adults or high-risk groups, showing alcohol use contributing to riskier sexual choices. Adults now in early midlife have been exposed to heavier drinking norms than previously, raising questions about effects on sexual well-being. We examined self-reported use and consequences of alcohol in sexual contexts, and its association with usual drinking pattern at age 38, and also associations of heavy drinking occasion (HDO) frequency with number of sexual partners, sexually transmitted infection (STI), and termination of pregnancy (TOP), from 2632 and 32-38 years of age.

Methods Members of the Dunedin Study birth cohort answered computer-presented questions about sexual behaviour and 